\title{
Gray Matter Abnormalities in Social Anxiety Disorder: Primary, Replication, and Specificity Studies
}

\author{
Ardesheer Talati, Spiro P. Pantazatos, Franklin R. Schneier, Myrna M. Weissman, and Joy Hirsch
}

Background: Despite increasing evidence that neuroanatomical abnormalities underlie pathological anxiety, social anxiety disorder (SAD)-although among the most common of anxiety disorders- has received little attention. With magnetic resonance imaging, we: 1) examined gray matter (GM) differences between generalized SAD and healthy control groups; 2) retested the findings in an independent clinical sample; and 3) tested for specificity by contrasting the SAD group to a separate group of panic disorder (PD) subjects.

Methods: The primary SAD group $(n=16)$ was required to meet DSM-IV criteria for SAD, with onset by age 30 years; control subjects ( $n=$ 20) had no lifetime history of anxiety. The replication sample included 17 generalized SAD and 17 control subjects. The PD comparison group $(n=16)$ was required to have no lifetime SAD. Images were acquired on a 1.5-Tesla GE Signa magnetic resonance imaging scanner with a three-dimensional T1-weighted spoiled gradient recalled pulse sequence. Morphological differences were determined with voxel-based morphometry, in SPM8.

Results: After adjusting for age, gender, and total intracranial volume, SAD (as compared with control) subjects had greater GM in the left parahippocampal and middle occipital, and bilateral supramarginal and angular cortices, and left cerebellum; and lower GM in bilateral temporal poles and left lateral orbitofrontal cortex. Cerebellar, parahippocampal, and temporal pole differences were observed in both samples, survived whole brain corrections, and were not observed in the PD group, pointing to relative specificity to SAD.

Conclusions: These findings parallel the functional literature on SAD and suggest structural abnormalities underlying the functional disturbances.

\begin{abstract}
Key Words: Cerebellum, magnetic resonance imaging, panic disorder, parahippocampal gyrus, social anxiety disorder/social phobia, temporal pole, voxel-based morphometry (VBM)
\end{abstract}

A nxiety disorders, as defined by the current DSM-IV (1), are among the most common psychiatric disorders. They share prominent anxiety as a clinical feature as well as some abnormalities in brain circuitry associated with fear processing (2) Anxiety is also clinically heterogeneous $(3,4)$, however, and identifying abnormalities in brain structure and function that pertain to the different diagnoses might help our understanding of the bases of this heterogeneity. Social anxiety disorder (SAD), although among the most common of anxiety disorders (5), has received relatively little attention in this context.

Social anxiety disorder is characterized by significant and persistent fear of social situations wherein the individual might be exposed to unfamiliar persons or situations or to scrutiny by others (1). Lifetime prevalence is approximately $5 \%-12 \%$, with higher rates among female individuals, and with mean onset in late childhood/ early adolescence $(6,7)$. Persons suffering from SAD typically either avoid the feared situations or endure them with intense anxiety or distress, leading to significant functional impairment (8). Generalized SAD—-the subtype involving experience of fear

From the Departments of Psychiatry (AT, FRS, MMW), Physiology and Cellular Biophysics (SPP), Epidemiology (MMW), Psychology (JH), Neuroscience $(\mathrm{JH})$, and Radiology $(\mathrm{JH})$, Columbia University Medical Center; Divisions of Epidemiology (AT, MMW) and Clinical Therapeutics (FRS), New York State Psychiatric Institute; and the functional MRI Laboratory (SPP, JH), Columbia University, New York, New York.

Authors AT and SPP contributed equally to this work

Address correspondence to Ardesheer Talati, Ph.D., Department of Psychiatry, Columbia University Medical Center, New York State Psychiatric Institute, 1051 Riverside Drive, Unit 24, New York, NY 10032; E-mail: at2071@columbia.edu.

Received Mar 19, 2012; revised May 23, 2012; accepted May 24, 2012. and avoidance in multiple situations-is associated with greater severity, comorbidity, and impairment and might have greater genetic heritability $(9,10)$.

Imaging studies have reported hyperactivity within limbic regions in SAD patients, particularly the amygdala, hippocampal region, and insula, when viewing emotionally charged faces $(11,12)$. These paradigms have particular face-validity for SAD, where fear of scrutiny and negative evaluation and avoidance of eye contact are core symptoms (13). Disturbances in frontal and particularly anterior cingulate cortex have been reported as well, although specificity and directionality of findings have been inconsistent (11). Other functional paradigms targeting anticipation of public speaking $(14,15)$, gaze or eye contact $(16)$, and judgment of self- versus non-self-relevant information $(17,18)$ have yielded generally similar patterns.

Data from functional paradigms, however, are dependent on the type of task performed as well as the current state of the subject. This is of particular concern in studies of SAD, because the scanning environment might exacerbate performance anxiety-a common feature of the disorder-and impair task performance. Measures of brain structure, in contrast, are largely state-independent and can complement functional studies by identifying morphological vulnerabilities that are robust to task parameters. Structural studies of SAD, however, have been extremely limited. A 2008 review of structural imaging studies of anxiety (19) identified only one report for SAD (20). That example failed to detect any differences between SAD cases and control subjects but was restricted to examination of the thalamus, putamen, and an overall index of gray matter (GM) (20). A subsequent meta-analysis of anxiety disorders failed to find any studies of SAD that qualified for inclusion (21). Some studies have included SAD subjects within anxiety groups but without differentiating them from other fear-based disorders (22). Finally, a recent treatment study reported volume decreases in the cerebellum and superior temporal cortex in SAD patients after 12 weeks of treatment with the selective serotonin reuptake inhibitor (SSRI) escitalopram (23). The SSRIs, however, are broadly efficacious for multiple anxiety and mood disorders, so the extent to which the 
changes index social anxiety is unknown. These questions, coupled with the overall paucity of studies, invite additional investigation with complementary approaches and populations.

We used magnetic resonance imaging and voxel-based morphometry (VBM) to identify brain abnormalities associated with SAD. Given the absence of well-established structural abnormalities for the disorder, we used a whole-brain approach involving three stages. First, we compared a primary group of persons with DSM-IV generalized SAD with a group of healthy control participants, to identify GM differences between the two groups. We then re-examined the same measures in an independent clinical sample of generalized SAD patients and healthy control subjects, to replicate and evaluate the generalizability of our findings. And finally, we asked whether the abnormalities were specific to SAD, by contrasting the primary SAD group to subjects with a different anxiety disorder: panic disorder (PD). Panic disorder is a complex disorder characterized by recurrent episodes of unexpected and uncontrollable fear, accompanied by cardio-respiratory and other autonomic responses. Like SAD, it is more frequent among women and moderately heritable, although with later onset $(24,25)$. Although the two disorders share some clinical symptoms as well as abnormal fear circuitry $(26,27)$, they also have distinguishing clinical and treatment profiles. Comparison with the PD group thus afforded us one mechanism to evaluate whether the aforementioned regional abnormalities specifically indexed social anxiety.

The goals of the study can thus be summarized as follows: 1) to identify brain abnormalities associated with SAD; 2) to retest the findings in an independent clinical population; and 3) to test specificity of these findings to social anxiety.

\section{Methods and Materials}

\section{Primary Sample ("Sample 1")}

All subjects were $18-50$ years of age. The SAD cases were required to have a DSM-IV (1) diagnosis of generalized social anxiety disorder (28) with onset by age 30 years and have a first-degree relative with an anxiety disorder. Control subjects were required to have no lifetime history of any psychiatric disorder, with exceptions for past minor depressive disorder, adjustment disorders, or brief periods of substance abuse (not dependence) in adolescence or college. Control subjects could also not have a history of an anxiety disorder in any first-degree relative. Neither group could have a personal or family history of schizophrenia or bipolar disorder.

Subjects were recruited through web advertisements (except seven SAD subjects recruited from an ongoing genetic program project of anxiety [29]). Subjects responding to the advertisement were first screened by a research assistant with the anxiety screening modules of the Schedule for Affective Disorders and Schizophrenia-Lifetime Version, Modified for Anxiety Disorders and Updated for DSM-IV (SADS-LA-IV [30]); subjects who screened positive for SAD then participated in a full DSM-IV interview (see following text). Similar procedures were used for the PD group, except PD subjects could not have a lifetime diagnosis of SAD, and vice versa. All procedures were approved by the Columbia University/New York State Psychiatric Institute Institutional Review Boards, and all subjects provided written consent.

Diagnostic assessments were administered by clinically trained mental health professionals with the SADS-LA-IV (30). Training and monitoring procedures have been previously described (29). Family history was obtained with the Family History Screen (31). Final diagnoses were made by an experienced clinician with the Best Estimate Procedure (32). Trait and state anxiety were assessed just before the scan with the Spielberger State-Trait Anxiety Inventory (33).

\section{Sample 2}

Structural data were obtained for 17 SAD and 17 healthy control (20-52 years of age) subjects who were imaged as part of an unrelated study (F.R.S., Principal Investigator) (16) with the same scanner. Subjects were recruited through media advertisements and clinical referrals and interviewed with the Structured Clinical Interview for DSM-IV Axis 1 Disorders (34). Severity was also rated by a clinician with the Liebowitz Social Anxiety Scale (35). The SAD group was required to have current generalized SAD but no other current Axis I disorder (except secondary diagnoses of generalized anxiety, dysthymia, or specific phobia). Control subjects were required to have no lifetime history of any Axis I disorder. Only images acquired at baseline (at which time all subjects had been medication-free for $\geq 4$ weeks) were used.

\section{Imaging and Data Analysis}

Structural data were acquired on a 1.5-Tesla GE Signa magnetic resonance imaging scanner (General Electric, Milwaukee, Wisconsin) with a three-dimensional T1-weighted spoiled gradient recalled pulse sequence with isomorphic voxels $(1 \times 1 \times 1$ $\mathrm{mm})$ in a $24-\mathrm{cm}$ field of view ( $256 \times 256$ matrix; approximately 186 slices; repetition time: $34 \mathrm{msec}$; echo time: $3 \mathrm{msec})$. Anatomical data were processed with whole-brain VBM $(36,37)$, implemented in SPM8 software (http://www.fil.ion.ucl.ac.uk/spm) with Matlab (version 7.13, Mathworks, Natick, Massachusetts). Three-dimensional T1-weighted images were segmented into the three main tissue classes (GM, white matter [WM], and cerebrospinal fluid) with the SPM unified segmentation algorithm (38). The GM and WM images were next spatially normalized to a group specific template and then to Montreal Neurological Institute space with a diffeomorphic image registration toolkit (Diffeomorphic Anatomical Registration using Exponentiated Lie algebra) in 1.5-mm cubic resolution (39). The images were modulated with the individual Jacobian determinants to preserve the local amount of GM and WM (40). Modulation was achieved by multiplying voxel values in the segmented images by the Jacobian determinants derived from the spatial normalization step. In effect, the analysis of modulated data tests for regional differences in the absolute amount of GM. Finally, images were smoothed with an 8-mm full-width-at-half-maximum isotropic Gaussian kernel. This is the SPM default, optimal for group inference (41), and commonly used in studies of anxiety (e.g., $[22,42,43])$, aiding future comparisons of our data with other studies.

Before statistical analysis, an inclusion mask was created by absolute thresholding, which excluded all voxels with $G M$ values $<$.2. Statistical analysis on processed GM images was carried out by means of whole brain multiple regression, with binary variables to code for SAD cases versus control subjects. Gender, age, and total intracranial volume (TIV) (which was the sum of GM, $W M$, and cerebrospinal fluid, for each subject normalized by $10,000)$ were always entered as covariates, because these are independently associated with GM differences in adults, and failure to adjust for these variables can result in false positives (44). For the combined dataset analysis, an additional variable coding for dataset was included to control for any possible systematic differences between samples. For whole-brain analyses, maps were thresholded at $p=.001$ and cluster-size of 10 (45). Additionally, significant clusters were identified by means of nonstationary cluster extent correction with random fields (46) 
Table 1. Sample Demographic Data and Clinical Features

\begin{tabular}{|c|c|c|c|c|c|c|}
\hline \multirow[b]{2}{*}{ Sample 1} & \multicolumn{3}{|c|}{ Diagnostic Groups } & \multicolumn{3}{|c|}{ Statistical Comparisons } \\
\hline & $\begin{array}{c}\text { SAD } \\
(n=16)\end{array}$ & $\begin{array}{c}\text { CON } \\
(n=20)\end{array}$ & $\begin{array}{c}\text { PD } \\
(n=16)\end{array}$ & SAD vs. CON & PD vs. CON & SAD vs. PD \\
\hline Gender (female) & $13(81)$ & $9(45)$ & $13(76)$ & $\chi^{2}=4.9^{a}$ & $\chi^{2}=3.8^{b}$ & $\chi^{2}=.11$ \\
\hline Age, mean yrs (SD) & $34.1(6.7)$ & $31.4(7.8)$ & $31.8(10)$ & $t=1.1$ & $t=.41$ & $t=.75$ \\
\hline Education (completed college) & $11(69)$ & $14(70)$ & $9(56)$ & $\chi^{2}=.007$ & $\chi^{2}=.72$ & $x^{2}=.53$ \\
\hline Age at Onset, mean (SD) & $11.0(5.9)$ & $\mathrm{n} / \mathrm{a}$ & $18.4(3.4)$ & & & $t=4.4^{c}$ \\
\hline Trait Anxiety, mean (SD) & $35.7(12.5)$ & $27(6.1)$ & $39.4(7.9)$ & $t=2.3^{a}$ & $t=4.9^{c}$ & $t=.99$ \\
\hline State Anxiety, mean (SD) & $39(11.9)$ & $26(4.1)$ & $35.5(10)$ & $t=4.0^{c}$ & $t=3.5^{d}$ & $t=.88$ \\
\hline \multicolumn{7}{|l|}{ Lifetime Comorbid Diagnoses } \\
\hline MDD & $5(31)$ & 0 & $3(17)$ & & & $\chi^{2}=.83$ \\
\hline GAD & $2(12)$ & 0 & $2(12)$ & & & $\chi^{2}=.004$ \\
\hline $\mathrm{SP}$ & $4(25)$ & 0 & $5(29)$ & & & $\chi^{2}=.08$ \\
\hline OCD & $1(6)$ & 0 & $2(12)$ & & & $\chi^{2}=.1$ \\
\hline DUD & $1(6)$ & 0 & $4(23)$ & & & $\chi^{2}=1.9$ \\
\hline AUD & 0 & 0 & $4(34)$ & & & $\chi^{2}=.3$ \\
\hline Lifetime Psych. Medication Use & $3(20)$ & 0 & $9(52)$ & & & $x^{2}=3.7^{b}$ \\
\hline Sample 2 & $\begin{array}{c}\text { SAD } \\
(n=17)\end{array}$ & $\begin{array}{c}\text { CON } \\
(n=17)\end{array}$ & $\begin{array}{l}\mathrm{PD} \\
\mathrm{n} / \mathrm{a}\end{array}$ & SAD vs. CON & & \\
\hline Gender (female) & $11(64)$ & $10(59)$ & & $x^{2}=.1$ & & \\
\hline Age, mean yrs (SD) & $29.1(8.9)$ & $31.3(10.7)$ & & $t=.66$ & & \\
\hline Education (completed college) & $12(71)$ & $10(60)$ & & $\mathrm{x}^{2}=.52$ & & \\
\hline State Anxiety, mean (SD) & $44.7(9.6)$ & $24.4(7.1)$ & & $t=6.9^{c}$ & & \\
\hline Liebowitz Social Anxiety Scale, mean (SD) & $81.4(15.6)$ & $8.1(5.4)$ & & $t=17.7^{c}$ & & \\
\hline \multicolumn{7}{|l|}{ Lifetime Comorbid Diagnoses } \\
\hline MDD & $6(35)$ & 0 & & & & \\
\hline GAD & $3(18)$ & 0 & & & & \\
\hline AUD & $2(12)$ & 0 & & & & \\
\hline Lifetime Psych. Medication Use & $6(35)$ & $2(12)$ & & & & \\
\hline Combined Sample & $\begin{array}{c}\text { SAD } \\
(n=33)\end{array}$ & $\begin{array}{c}\text { CON } \\
(n=37)\end{array}$ & $\begin{array}{l}\mathrm{PD} \\
\mathrm{n} / \mathrm{a}\end{array}$ & $\begin{array}{l}\text { Sample } \\
\text { (1 vs. } 2 \text { ) }\end{array}$ & $\begin{array}{c}\text { Group } \\
\text { (SAD vs. CON) }\end{array}$ & $\begin{array}{c}\text { Sample x } \\
\text { Group }\end{array}$ \\
\hline Gender (female) & $24(73)$ & $19(51)$ & & $x^{2}=.03$ & $x^{2}=3.4^{b}$ & $x^{2}=1.8$ \\
\hline Age, mean yrs (SD) & $31.5(8.2)$ & $31.4(9.1)$ & & $t=1.3$ & $t=.01$ & $F=1.5$ \\
\hline Education (completed college) & $23(70)$ & $24(65)$ & & $x^{2}=.3$ & $\mathrm{x}^{2}=.18$ & $x^{2}=.01$ \\
\hline State Anxiety, mean (SD) & $42.0(11.0)$ & $25.6(5.7)$ & & $t=1.5$ & $t=64.9^{c}$ & $F=2.8$ \\
\hline
\end{tabular}

Values given are number (\%), unless otherwise indicated. Medication frequencies only include those prescribed for a psychiatric condition.

AUD, alcohol use disorder (abuse or dependence); CON, control subjects; DUD, drug use disorder (abuse or dependence); GAD, generalized anxiety disorder; $\mathrm{MDD}$, major depressive disorder; $\mathrm{OCD}$, obsessive compulsive disorder; PD, panic disorder; $\mathrm{SAD}$, social anxiety disorder; $\mathrm{SP}$, specific phobia.

${ }^{a} p \leq .05$.

${ }^{b} p \leq .1$.

${ }^{c} p \leq .001$

${ }^{d} p \leq .005$

as implemented with the NS toolbox (http://fmri.wfubmc.edu/ $\mathrm{cms}$ /software\#NS) for SPM5. This correction method confers increased sensitivity to spatially extended signals while remaining valid when cluster-size distribution varies, depending on local smoothness as is the case in VBM data (46).

\section{Results}

\section{Sample 1}

Demographic and Clinical Features. Sample characteristics are detailed in Table 1 (Sample 1). As compared with the control subjects, the SAD group had a higher proportion of female subjects and reported higher state and trait anxiety. The groups did not differ by age or education. The most frequently comorbid lifetime diagnoses were major depressive disorder and specific phobia. Three subjects reported taking medication for anxiety in the past, but no subject was taking any psychoactive medication in the 10 weeks preceding the scan.

GM Differences Associated with SAD. We first examined GM differences between the SAD and healthy control groups across the entire brain. Significant group differences (10 or more voxels, $p \leq$ .001 ) are identified in Table 2 (Sample 1). All analyses were adjusted for age, gender, and TIV. There were no overall differences in total gray or white matter between the SAD and control groups.

The largest GM increases associated with SAD were in a left hemisphere cluster encompassing the cerebellum and fusiform/ parahippocampal cortex (Brodmann areas [BAs] 37 and 36). Addi- 
Table 2. Gray Matter Abnormalities Associated with SAD

\begin{tabular}{|c|c|c|c|c|c|c|c|}
\hline & & BA & Size & $\mathrm{x}$ & $y$ & $z$ & $t$ \\
\hline \multicolumn{8}{|c|}{ Sample 1} \\
\hline \multicolumn{8}{|c|}{ SAD $>$ Control } \\
\hline 1 & L cerebellum, parahippocampal, fusiform & 37,36 & $451^{a}$ & -24 & -39 & -21 & 4.40 \\
\hline 2 & R middle frontal & 46,10 & 224 & 45 & 51 & 9 & 4.95 \\
\hline 3 & R lingual & 19 & 41 & 24 & -53 & -5 & 3.67 \\
\hline 4 & $\mathrm{R}$ cerebellum & - & 35 & 15 & -62 & -44 & 3.95 \\
\hline 5 & L middle occipital & 19 & 11 & -39 & -74 & 8 & 3.98 \\
\hline 6 & L lingual & 17 & 12 & -8 & -92 & -15 & 3.49 \\
\hline \multicolumn{8}{|c|}{ Control $>$ SAD } \\
\hline 1 & R precentral, postcentral & 6,4 & $678^{b}$ & 42 & -18 & 38 & -4.84 \\
\hline 2 & $\mathrm{R}$ middle cingulate & 24 & 199 & 14 & -21 & 48 & -4.64 \\
\hline 3 & L middle cingulate & 32 & 32 & -11 & 21 & 38 & -3.89 \\
\hline 4 & L superior temporal & 22 & 29 & -59 & 8 & 3 & -4.13 \\
\hline 5 & $\mathrm{R}$ temporal pole, superior temporal & 38 & 28 & 30 & 17 & -29 & -3.36 \\
\hline 6 & $\mathrm{R}$ medial frontal, middle cingulate & 6,24 & 16 & 11 & -6 & 51 & -4.47 \\
\hline 7 & L middle cingulate & 24 & 11 & -14 & -20 & 44 & -3.59 \\
\hline \multicolumn{8}{|c|}{ Sample 2} \\
\hline \multicolumn{8}{|c|}{ SAD $>$ Control } \\
\hline 1 & $\mathrm{~L}, \mathrm{R}$ cerebellum & - & 701 & 2 & -41 & -12 & 4.92 \\
\hline 2 & L inferior parietal, supramarginal & 40 & 214 & -38 & -42 & 53 & 3.92 \\
\hline 3 & R paracentral lobule, supp. motor area & 6 & 186 & 5 & -17 & 48 & 4.24 \\
\hline 4 & L inferior temporal & 20,21 & 153 & -57 & -44 & -14 & 4.97 \\
\hline 5 & $\mathrm{R}$ post central gyrus & $3,1,2$ & 29 & 39 & -24 & 48 & 3.95 \\
\hline \multicolumn{8}{|c|}{ Control > SAD } \\
\hline 1 & R temporal pole, superior temporal & 38 & $603^{b}$ & 38 & 17 & -29 & -4.91 \\
\hline 2 & $\mathrm{R}$ middle frontal, orbitofrontal & 11,47 & $366^{a}$ & 33 & 47 & -9 & -5.66 \\
\hline 3 & L temporal pole, superior temporal & 38 & 31 & -42 & 20 & -27 & -3.71 \\
\hline 4 & L inferior frontal, orbitofrontal & 11 & 22 & -36 & 33 & -9 & -4.14 \\
\hline \multicolumn{8}{|c|}{ Combined Sample } \\
\hline \multicolumn{8}{|c|}{ SAD $>$ Control } \\
\hline 1 & L cerebellum, parahippocampal, fusiform & 37 & $1840^{b}$ & 0 & -51 & -12 & 4.12 \\
\hline 2 & R supramarginal, angular & 40 & 192 & 53 & -50 & 36 & 3.81 \\
\hline 3 & L supramarginal, angular & 40 & 22 & -42 & -62 & 47 & 3.44 \\
\hline 4 & L middle occipital & 19 & 28 & -39 & -71 & 8 & 3.73 \\
\hline \multicolumn{8}{|c|}{ Control $>$ SAD } \\
\hline 1 & $\mathrm{R}$ temporal pole, superior temporal & 38 & $851^{b}$ & 32 & 17 & -30 & -5.22 \\
\hline 2 & L temporal pole, superior temporal & 38 & 97 & -42 & 15 & -30 & -3.56 \\
\hline 3 & L inferior frontal, orbitofrontal & 47 & 18 & -38 & 35 & -9 & -3.69 \\
\hline 4 & R superior occipital & & 10 & 24 & -74 & 29 & -3.55 \\
\hline
\end{tabular}

Clusters are listed in order of descending size; coordinates refer to the voxel with the peak t value in the cluster; $p<$ $.001 ; \mathrm{k}=10$. Sample 1: $n=36$ (16 SAD, 20 Control); Sample 2: $n=34$ (17 SAD, 17 Control); Combined Sample: $n=70$

(33 SAD, 37 Control).

BA, Brodmann area; L, left; R, right; supp., supplementary; other abbreviations as in Table 1.

Clusters surviving whole brain correction are indicated as follows.

${ }^{a} p<.1$.

${ }^{b} p<.05$.

tional differences were detected in right and left lingual, middle occipital, and middle frontal gyri. The converse contrast (control > SAD) identified a cluster spanning right hemisphere primary motor and sensory cortices, multiple clusters in both hemispheres of the dorsal anterior cingulate, and a cluster in the temporopolar region of the left superior temporal cortex.

\section{Sample 2}

Demographic and Clinical Features. We next repeated the aforementioned analyses in an independently recruited and imaged clinical sample of SAD cases and healthy control subjects (sample 2). The SAD and control groups of sample 2 did not significantly differ on measures of age, gender, or education, either from each other (Table 1, Sample 2) or from the corresponding groups in the first sample (Table 1, Combined Sample).
GM Differences Associated with SAD. The GM differences between the sample 2 SAD and control groups are listed in Table 2 (Sample 2), adjusted for age, gender, and TIV. Significantly greater GM among the SAD group was detected in the bilateral cerebellum, left supramarginal, right paracentral lobule and supplementary motor area, left inferior temporal, and right post-central regions. The control $>$ SAD contrast identified clusters in bilateral temporal pole and regions of the middle and inferior frontal gyri encompassing orbitofrontal cortex (OFC). Similar clusters were identified when using an alternative continuous measure of social anxiety rather than a diagnosis (Supplement 1).

\section{Combined Sample}

We next combined the two samples into a single dataset and examined overall differences between SAD and control groups, 


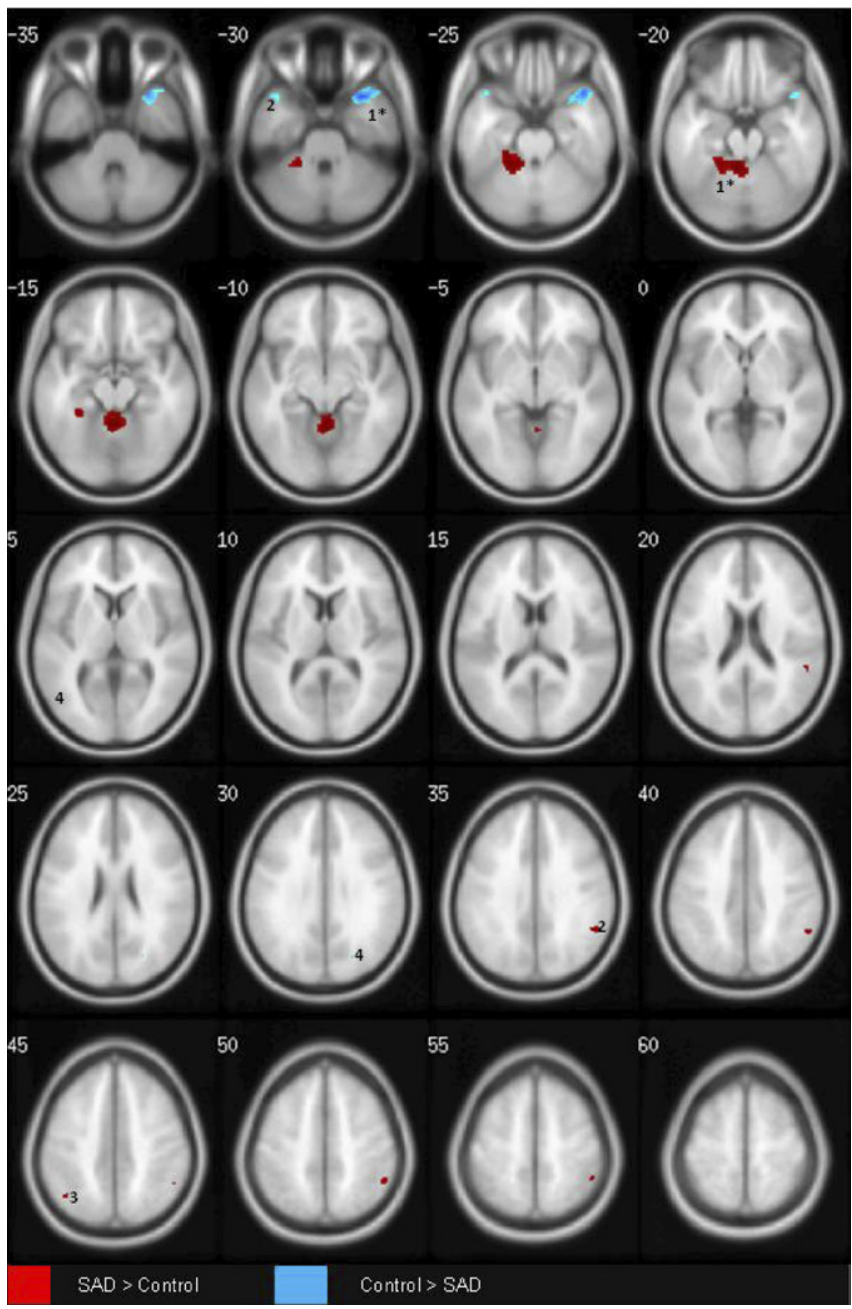

Figure 1. Gray matter differences associated with social anxiety disorder (SAD); $p \leq .001 ; \mathrm{k}=10 ; n=70$ (33 SAD, 37 Control). T1-weighted axial images; image left is brain left. Images Group differences are adjusted for differences in age, gender, intracranial volume, and sample source. *Regions surviving multiple comparison correction at the whole brain level. Clusters are numbered corresponding to their listing in Table 2 (Combined Sample). SAD > Control (red): 1: left (L) cerebellum, parahippocampal, fusiform; 2: right (R) supramarginal, angular; $3: \mathrm{L}$ supramarginal, angular; $4: \mathrm{L}$ middle occipital. Contro $>$ SAD (blue): 1: R superior temporal, anterior temporal pole; 2: $L$ superior temporal, anterior temporal pole; 3 ; L inferior frontal (orbitofrontal); $4: \mathrm{L}$ middle occipital.

adjusting for the previously noted variables as well as for sample of origin. The final results, detailed in Table 2 (Combined Sample), preserve a number of the regions observed in the individual samples. Specifically, the SAD > control contrast revealed large increases in the cerebellum, left parahippocampal and fusiform gyri, bilateral supramarginal and angular gyri, and left middle occipital gyrus. The control > SAD contrast identified lower temporal pole (both hemispheres, but predominantly right) and left inferior prefrontal/orbitofrontal GM in the SAD group. Cerebellum, parahippocampal, and temporal pole differences were robust to multiple comparison correction at the whole-brain level (asterisked clusters). The main findings are illustrated in Figure 1, with clusters shown in red illustrating regions with greater GM volume among the SAD group than the control subjects, and clusters in blue illustrating the converse. The numbering of regions in the figures corresponds to the clusters in Table 2 . Importantly, group differences in the cerebellum/parahippocampal gyrus (PHG) and temporal pole remained significant when we reran the aforementioned analysis with the same statistical thresholds and control subjects but only including the subgroup of SAD subjects who had any history of any other psychiatric disorder over their lifetime. This further minimizes the possibility that neuroanatomical variation related to other lifetime anxiety disorders could have contributed to the observed findings. Additional corollary analyses further confirmed that regional findings from one sample were replicated in the other (Supplement 1). Finally, an exploratory analysis of regions of interest that did not meet a priori criteria suggested lower right amygdala, bilateral insula, and left anterior cingulate GM in the SAD group (Supplement 1).

\section{Testing for Specificity}

To further investigate whether the GM differences identified in the preceding text were specific to $S A D$, we compared the SAD group from Sample 1 with a group of separate subjects from sample 1 with PD. The PD group and SAD groups did not differ on measures of age, gender, state or trait anxiety, or education (Table 1, Sample $1)$, although age of onset for PD was later (18 vs. 11 years), consistent with the epidemiology of the disorders.

We first examined differences between the PD and the control groups. As shown in Table 3 (PD vs. Control) and Figure 2A, subjects with PD, as compared with control subjects, had large parietooccipital GM increases-specifically, in bilateral cuneate and precuneate, lingual, and superior occipital cortices-as well as larger insular cortex. Conversely, a number of frontal cortical (right preand post-central gyri, left and right middle cingulate, supplementary motor area) as well as subcortical (thalamus, caudate) regions showed reduced GM among the PD cases.

We then formally contrasted the PD and SAD groups. As shown in Table 3 (PD vs. SAD) and Figure 2B, subjects with PD showed larger mean occipital GM volume, particularly in the cuneate cortex, calcarine sulcus, and lingual gyrus. Conversely, both hemispheres of the parahippocampal and fusiform gyri were significantly larger in the SAD group. Finally, right inferior frontal (orbitofrontal), and anterior cingulate were larger among the SAD groups, although both anxiety groups had reduced GM vis-a-vis healthy control subjects.

\section{Discussion}

\section{Summary of Findings}

We report here on morphological abnormalities associated with generalized SAD. We found that subjects with the disorder, as compared with healthy control subjects, had greater GM in the cerebellum and the left parahippocampal cortex and lower GM in the temporal pole. Several observations strengthen our confidence in these findings. First, the differences were observed in both individual samples as well as in the combined dataset analysis. Second, the clusters remained significant after correction for multiple comparisons. Third, similar findings were obtained when using a severity index rather than a diagnosis of SAD (sample 2). And finally, subjects with panic disorder (PD) did not show these patterns, pointing to the relative specificity of these findings to SAD. We thus weight the ensuing discussion primarily toward the aforementioned regions. Other GM differences that were not observed in both samples or did not survive corrections for multiple comparisons might play a role, but such findings should be considered provisional.

\section{GM Increases Associated with SAD}

The largest differences were detected within the cerebellum. Resting state perfusion studies have reported both hyper- and hy- 
Table 3. Gray Matter Differences Between Panic Disorder and Social Anxiety Disorder

\begin{tabular}{|c|c|c|c|c|c|c|c|}
\hline & & BA & Size & $\mathrm{x}$ & y & z & $t$ \\
\hline \multicolumn{8}{|c|}{ PD vs. Control ${ }^{a}$} \\
\hline \multicolumn{8}{|c|}{ PD $>$ Control } \\
\hline 1 & $L, R$ cuneus, lingual & 17,18 & $1620^{b}$ & 0 & -98 & 6 & 5.03 \\
\hline 2 & L Insula & - & 379 & -30 & 9 & 5 & 4.44 \\
\hline 3 & $L, R$ cuneus, precuneus & 7 & 355 & 0 & -71 & 24 & 4.30 \\
\hline 4 & R cuneus, superior occipital & 7 & 23 & 21 & -75 & 30 & 3.56 \\
\hline \multicolumn{8}{|c|}{ Control $>$ PD } \\
\hline 1 & R precentral, postcentral & $6,1-4$ & $884^{b}$ & 44 & -14 & 42 & -5.29 \\
\hline 2 & $\mathrm{R}$ middle cingulate & 32 & $504^{b}$ & 5 & 20 & 41 & -4.69 \\
\hline 3 & L inferior parietal & 40 & 104 & -50 & -45 & 42 & -4.36 \\
\hline 4 & $\mathrm{R}$ middle cingulate, $\mathrm{R}$ supp. motor area & 24 & 29 & 11 & -6 & 50 & -4.32 \\
\hline 5 & L caudate & - & 98 & -14 & 12 & 18 & -4.14 \\
\hline 6 & L precentral & 6 & 40 & -56 & -5 & 36 & -3.64 \\
\hline 7 & $\mathrm{R}$ middle cingulate & 24 & 13 & 15 & -18 & 44 & -3.57 \\
\hline 8 & $\mathrm{R}$ thalamus & - & 23 & 8 & -20 & 15 & -3.46 \\
\hline \multicolumn{8}{|c|}{ PD vs. SAD ${ }^{c}$} \\
\hline \multicolumn{8}{|c|}{$P D>S A D$} \\
\hline 1 & L cuneus & 7 & 149 & -6 & -77 & 36 & 4.43 \\
\hline 2 & L middle frontal & 9 & 35 & -53 & 20 & 30 & 4.19 \\
\hline 3 & $\mathrm{~L}, \mathrm{R}$ lingual & 18 & 25 & 2 & -87 & -8 & 3.56 \\
\hline 4 & R superior occipital & 18,31 & 12 & 23 & -74 & 29 & 3.74 \\
\hline \multicolumn{8}{|c|}{$S A D>P D$} \\
\hline 1 & $\mathrm{R}$ parahippocampal, fusiform, cerebellum & $37,36,19$ & $534^{b}$ & 30 & -48 & -11 & -4.52 \\
\hline 2 & L parahippocampal, fusiform & 37,36 & 402 & -30 & -41 & -17 & -4.42 \\
\hline 3 & $\mathrm{R}$ middle frontal, inferior frontal & 10 & 106 & 42 & 45 & 5 & -3.94 \\
\hline 4 & R anterior cingulate & 32 & 10 & 15 & 47 & 2 & -3.55 \\
\hline 5 & L middle frontal & 8 & 12 & -21 & 21 & 44 & -3.34 \\
\hline
\end{tabular}

Clusters are listed in order of descending size; coordinates refer to the voxel with the peak $t$ value in the cluster; $p<$ $.001 ; \mathrm{k}=10$

Abbreviations as in Tables 1 and 2

$a_{n}=16 \mathrm{PD}, n=20$ Control.

${ }^{b}$ Clusters surviving whole brain correction $(p<.05)$. $c_{n}=16 \mathrm{SAD}, n=16 \mathrm{PD}$.

po-perfusion in the cerebellum among subjects with SAD (47), and a positron emission tomography study found that anxiety induced in SAD patients increased blood flow to the cerebellum (48). The aforementioned treatment study (23) reported decreased cerebellar volumes among SAD patients after 3 months of SSRI treatment, but because there was no control group, it is unclear whether the patients had abnormalities before being treated. Although the mechanisms are unclear, cerebellar abnormalities might increase vulnerability to anxiety states via modulation of arousal. Many cerebellar subdivisions and the vermis in particular project to the midbrain regions of the pons and medulla, which mediate the autonomic responses that are exaggerated in persons with anxiety (49).

Also having higher GM volume in the SAD group was the PHG. The PHG consolidates memories and social communication cues, and hyperactivation has been reported in SAD patients during conditions of social threat $(50,51)$ as well as during nonthreatening tasks involving human as compared with nonhuman or computersimulated interaction (52). Moreover, the adjacent fusiform gyruspart of the parahippocampal cortex (and included in the detected clusters) - is cardinal in facial recognition (53) and processing of facial expression $(51,54)$. A recent functional magnetic resonance imaging study reported that, when asked to passively view socially threatening stimuli, persons with SAD had higher blood oxygen level-dependent signal increases in bilateral PHG than control subjects; however, if asked to try to actively regulate their negative responses to the same stimuli, the SAD group had decreased responses in fusiform (50). These differences were not replicated if social threat was replaced with physical threat. In the present study, PHG and fusiform GM differences were observed only in the SAD group (Table 3, PD vs. Control). The GM volume in the PD group was not only significantly lower than in the SAD group, as shown in Table 3 (PD vs. SAD), but lower than in the control subjects as well. Although no other study to our knowledge has directly contrasted these two anxiety disorders at the morphological level, a number of reports (55-57) have reported reduced PHG volume among panic patients. Our data, coupled with the functional literature on SAD, suggest that increased PHG activity might serve as a marker for social-based anxiety constructs. Incidentally, lower caudate volume, the only other regional abnormality associated with PD in the aforementioned PD meta-analysis (55), was also replicated in our PD group (Table 3, PD vs. Control).

\section{GM Decreases Associated with SAD}

The temporal pole (i.e., the anterior region of the superior temporal cortex corresponding to BA38) had significantly lower GM volume in both samples of SAD subjects. The anterior temporal cortex has been implicated in the processing of abstract conceptual knowledge, but BA38 - and particularly its right hemispheremight more specifically index social concepts $(58,59)$. Studies in healthy subjects have reported activation in this region during social competition and perception of the mental states of others (52). Conversely, lesions and degeneration of BA38 have led to 

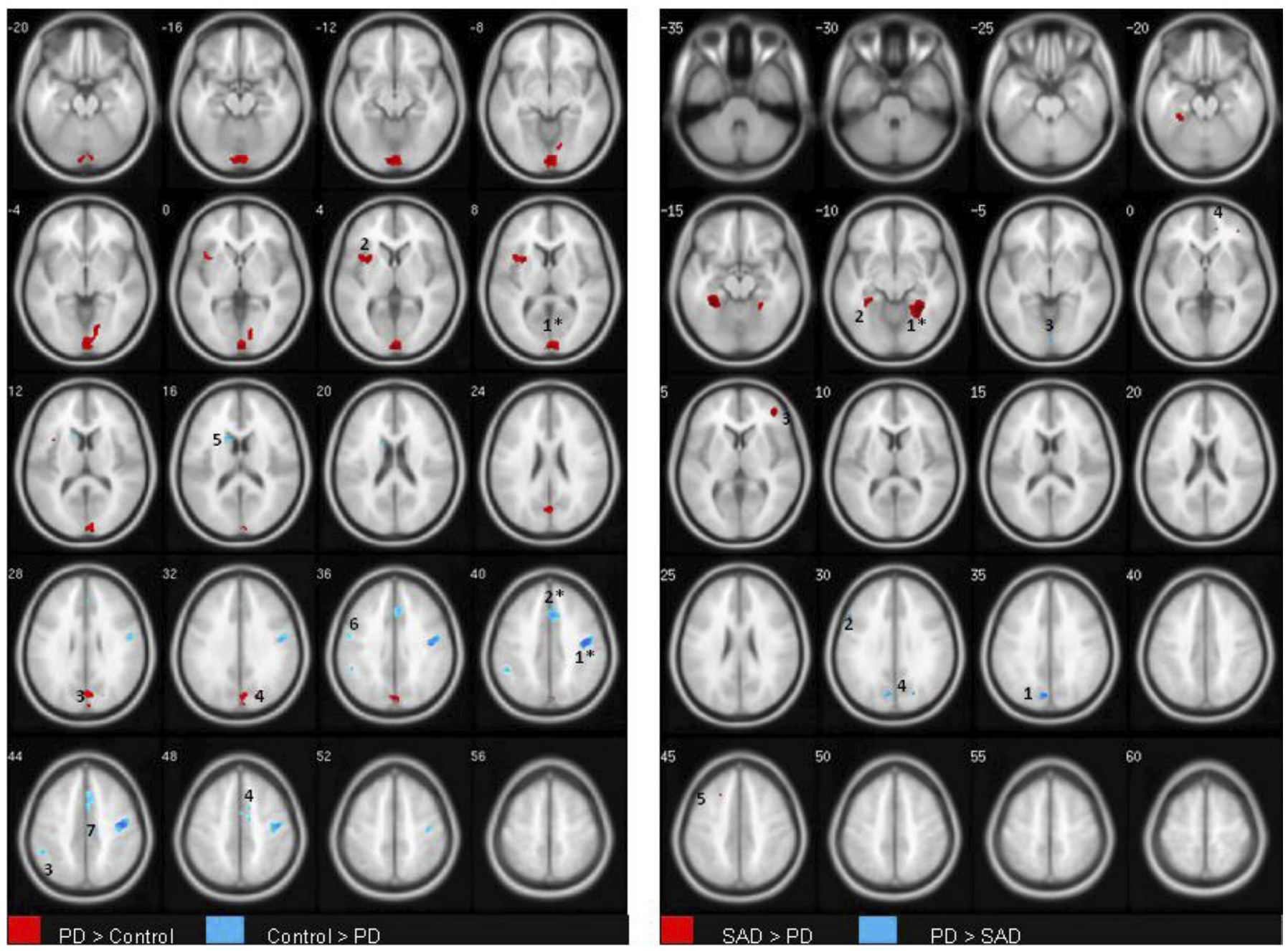

Figure 2. Gray matter differences between social anxiety disorder (SAD) and panic disorder (PD); $p \leq .001 ; \mathrm{k}=10$. (A) $n=16 \mathrm{PD}, n=20$ Control; (B) $n=16$ SAD, $n=16$ PD. T1-weighted axial images; image left is brain left. Images Group differences are adjusted for differences in age, gender, and intracranial volume. Clusters surviving whole brain correction are indicated as follows: ${ }^{*} p<.05 ;{ }^{+} p<1$. Clusters are numbered corresponding to their listings in Table 3 , PD vs. Control and PD. vs. Sad, respectively. (A) PD > Control (red): 1: bilateral cuneate, lingual; 2: L insula; 3: bilateral cuneus, precuneus; 4: R cuneus, superior occipital. Control > PD (blue) 1: R precentral, postcentral; 2: R middle cingulate; $3: \mathrm{L}$ inferior parietal; 4: $\mathrm{R}$ middle cingulate, supplementary motor area; 5 : $\mathrm{L}$ caudate; 6: precentral; 7: R middle cingulate. (B) SAD > PD (red): 1: R parahippocampal, fusiform; 2: L parahippocampal, fusiform; 3: R middle frontal, inferior frontal; 4: $R$ anterior cingulate; 5: $L$ middle frontal. $P D>S A D$ (blue) $1: L$ cuneus; $2: L$ middle frontal; 3 ; $L$ and $R$ lingual; 4: $R$ superior occipital.

changes in the ability to characterize social attributes of behavior $(58,60)$. Among persons with SAD, public speaking (15) and anticipation thereof (61) have been associated with decreased blood flow to the temporal pole. Interestingly, selectively increased surface area in the left temporal pole has been reported in Williams Syndrome (WS), a rare genetic disorder that in terms of its behavioral phenotype seems the opposite of SAD (e.g., hyper-sociability, lack of fear of interacting with strangers) (62). That, as well as one other report (63) also showed decreased PHG volume in WS patients, again the opposite of what we find here with SAD. Although WS and SAD might be etiologically different disorders, the common regional focus of abnormalities suggests that the aforementioned regions might mediate common constructs of social cognition, with different neuroanatomical aberrations leading to different clinical syndromes.

The SAD group also had lower GM in the lateral OFC (approximately BA11, BA47). The OFC regulates expression of emotion and assigns positive and negative stimulus response contingencies $(64,65)$. Concordantly, GM disturbances (particularly in the left hemisphere) have been linked to multiple anxiety and mood problems (18). The OFC also receives direct reciprocal input from the amygdala (47), a central mediator of the fear response, and in persons with SAD, the uncinate fasiculus - the white matter tract connecting OFC to temporal cortex-is compromised $(66,67)$. Although our study does not address temporal sequence, these disturbances are likely to begin early in life, because infants with high-reactive and inhibited temperament-which are risk factors for later onset of SAD $(68,69)$ - show reduced cortical thickness in similar left OFC regions when imaged in adulthood, even if they did not go on to develop the full disorder (70).

Finally, two other interesting but inconsistently observed regional differences deserve comment. First, significantly lower GM among SAD cases was identified in the primary motor and sensory cortex in sample 1 only. Although the right hemispheric precentral 
gyrus is thought to control motor function, it also has been associated with self-face recognition (48) and imitations of facial expressions (49) that could hold implications for social anxiety. Second, multiple clusters were observed for the control $>$ SAD contrast in the middle cingulate in sample 1 (Table 2). Although not directly mirrored in sample 2, within the SAD cases of sample 2, increased severity of social anxiety was associated with lower cingulate GM. Although it is unlikely that these patterns are specific to SAD (they were observed in the PD group, and similar abnormalities have been reported for other anxiety and mood disorders [21]), the overall inverse relationships with GM-SAD diagnosis (sample 1) and GM-severity (sample 2) are broadly consistent with functional models positing anxiety as a failure of the frontal cortex to downregulate limbic activation (71).

\section{Study Limitations}

The reported findings should be interpreted within the context of the following limitations. First, the study is cross-sectional and does not therefore speak to the causal relationship between brain structure and diseased state, because the identified GM differences could either predispose to or be a result of the disorder. Disentangling causal from compensatory pathways would require more complex epidemiological approaches (72) (e.g., selecting subjects who are at high-risk [by virtue of family history, presence of a prodrome, and the like] but asymptomatic at recruitment, and then tracking brain changes longitudinally as a subset that goes on to develop the syndrome). In a related limitation, the GM differences should also not be used to make diagnostic inferences, because they are based on overall group differences and do not account for important individual brain, behavioral, and environmental variations that shape whether a given subject will have a diagnosis (73). Second, standard methodological limitations to VBM, particularly its vulnerability to normalization and smoothing errors $(37,74)$, apply here as well. Third, it is possible that gender-related variance partially contributed to observed group differences in sample 1 , where anxiety groups had a greater proportion of female subjects. We did, however, adjust for gender in all models, and furthermore, the main findings were replicated in the second sample-which was matched on gender-as well as in the final combined sample. Fourth, although other lifetime anxiety diagnoses did not contribute to the observed results, we cannot rule out that other behavioral traits that differed across the two groups but do not directly index SAD contributed to group differences. Finally, we only compared SAD with PD. Different patterns might have been observed with a different comparison group: for example, specific phobia might have yielded largely overlapping coordinates, but posttraumatic stress disorder diverged significantly, particularly within frontal regulatory regions (71). In a related limitation, we only included SAD subjects without a history of $P D$, and vice versa. This could have biased selection toward milder or less-generalizable cases. Alternative approaches would be to include a third group with both SAD and PD or to permit all comorbidity and then model the variance statistically. Both approaches, however, would have necessitated a substantially larger sample.

\section{Conclusions}

This report contributes to the currently limited literature on the neurobiology of SAD by identifying structural deficits that might predispose to functional abnormalities. The rigorous ascertainment criteria, retest in an independent sample, and comparison with PD strengthen both the reliability and the interpretability of our findings. The confirmation in a second sample is particularly valuable in an imaging context, given the preponderance of failures to repli- cate original reports (75), and should be considered in future designs whenever possible. Finally, because the results include regions (e.g., cerebellum, temporal pole) that are not among the primary nodes of fear circuitry, the validity of these regions as well as their specific roles in mediating constructs of SAD will require further investigation.

Sample 1 collection and ascertainment was funded by a National Alliance for Research on Schizophrenia and Depression Young Investigator Grant (AT, Principal Investigator [P.I.]); some cases were recruited from an ongoing National Institute of Mental Health (NIMH) Program Project Grant P01 MH60970 (Rene Hen, Ph.D., Overall P.I.; MMW, Ph.D., Project P.I.). Brain scans were funded by the functional MRI Laboratory at Columbia University (JH, P.I.). Sample 2 was funded by a NIMH separate grant $R 21 \mathrm{MH} 077976$ (FRS, P.I).

Dr. Talati is funded by a 5-year K01 Award (1 K01 DA029598) from the National Institute of Drug Abuse; Spiro Pantazatos is funded by an F31 award (F31MH088104-02) from the NIMH.

We express our gratitude to Christine Platzek, M.S., and Sandhya Rawal, B.A. candidate, for assistance with subject recruitment and data analysis, respectively.

In the past 2 years, Dr. Weissman has received royalties from the Oxford University Press, Perseus Press, the American Psychiatric Association Press, and MultiHealth Systems. Dr. Schneier has received an honorarium from GlaxoSmithKline for speaking at two conferences and royalties from Cambridge University Press and UpToDate. None of these present a conflict of interest with the present study. Drs. Talati, Pantazatos, and Hirsch report no biomedical financial interests or potential conflicts of interest.

\section{Supplementary material cited in this article is available online.}

1. American Psychiatric Association (1994): Diagnostic and Statistical Manual of Psychiatric Disorders. Washington, DC: American Psychiatric Association.

2. Delgado MR, Nearing KI, Ledoux JE, Phelps EA (2008): Neural circuitry underlying the regulation of conditioned fear and its relation to extinction. Neuron 59:829-838.

3. Bienvenu OJ, Samuels JF, Wuyek LA, Liang KY, Wang Y, Grados MA, et al. (2012): Is obsessive-compulsive disorder an anxiety disorder, and what, if any, are spectrum conditions? A family study perspective. Psychol Med 42:1-13.

4. Stein DJ, Craske MG, Friedman MJ, Phillips KA (2011): Meta-structure issues for the DSM-5: How do anxiety disorders, obsessive-compulsive and related disorders, post-traumatic disorders, and dissociative disorders fit together? Curr Psychiatry Rep 13:248-250.

5. Jefferys D (1997): Social phobia. The most common anxiety disorder. Aust Fam Physician 26:1061, 4-7.

6. Weissman MM, Bland RC, Canino GJ, Greenwald S, Lee CK, Newman SC, et al. (1996): The cross-national epidemiology of social phobia: A preliminary report. Int Clin Psychopharmacol 11(suppl 3):9-14.

7. Kessler RC, Chiu WT, Demler O, Merikangas KR, Walters EE (2005): Prevalence, severity, and comorbidity of 12-month DSM-IV disorders in the National Comorbidity Survey Replication. Arch Gen Psychiatry 62:617627.

8. Filho AS, Hetem LA, Ferrari MC, Trzesniak C, Martin-Santos R, Borduqui T, et al. (2010): Social anxiety disorder: What are we losing with the current diagnostic criteria? Acta Psychiatr Scand 121:216-226.

9. Schneier FR (2006): Clinical practice. Social anxiety disorder. N Engl J Med 355:1029-1036.

10. Stein MB, Chartier MJ, Hazen AL, Kozak MV, Tancer ME, Lander S, et al. (1998): A direct-interview family study of generalized social phobia. Am J Psychiatry 155:90-97.

11. Freitas-Ferrari MC, Hallak JE, Trzesniak C, Filho AS, Machado-de-Sousa JP, Chagas MH, et al. (2010): Neuroimaging in social anxiety disorder: A systematic review of the literature. Prog Neuropsychopharmacol Biol Psychiatry 34:565-580. 
12. Pietrini F, Godini L, Lazzeretti L, Benni L, Pracucci C, Talamba GA, et al. (2010): [Neuroimaging and neurobiology of social anxiety]. Riv Psichiatr 45:349-360.

13. Safren SA, Heimberg RG, Horner KJ, Juster HR, Schneier FR, Liebowitz MR (1999): Factor structure of social fears: The Liebowitz Social Anxiety Scale. J Anxiety Disord 13:253-270.

14. Lorberbaum JP, Kose S, Johnson MR, Arana GW, Sullivan LK, Hamner MB, et al. (2004): Neural correlates of speech anticipatory anxiety in generalized social phobia. Neuroreport 15:2701-2705.

15. Tillfors M, Furmark T, Marteinsdottir I, Fischer H, Pissiota A, Langstrom B, et al. (2001): Cerebral blood flow in subjects with social phobia during stressful speaking tasks: A PET study. Am J Psychiatry 158:1220-1226.

16. Schneier FR, Pomplun M, Sy M, Hirsch J (2011): Neural response to eye contact and paroxetine treatment in generalized social anxiety disorder. Psychiatry Res 194:271-278.

17. Andrews-Hanna JR, Reidler JS, Sepulcre J, Poulin R, Buckner RL (2010): Functional-anatomic fractionation of the brain's default network. Neuron 65:550-562.

18. Whitfield-Gabrieli S, Moran JM, Nieto-Castanon A, Triantafyllou C, Saxe R, Gabrieli JD (2011): Associations and dissociations between default and self-reference networks in the human brain. Neuroimage 55:225232

19. Ferrari MC, Busatto GF, McGuire PK, Crippa JA (2008): Structural magnetic resonance imaging in anxiety disorders: An update of research findings. Rev Bras Psiquiatr 30:251-264.

20. Potts NL, Davidson JR, Krishnan KR, Doraiswamy PM (1994): Magnetic resonance imaging in social phobia. Psychiatry Res 52:35-42.

21. Radua J, van den Heuvel OA, Surguladze S, Mataix-Cols D (2010): Metaanalytical comparison of voxel-based morphometry studies in obses sive-compulsive disorder vs other anxiety disorders. Arch Gen Psychiatry 67:701-711.

22. van Tol MJ, van der Wee NJ, van den Heuvel OA, Nielen MM, Demenescu LR, Aleman A, et al. (2010): Regional brain volume in depression and anxiety disorders. Arch Gen Psychiatry 67:1002-1011.

23. Cassimjee N, Fouche JP, Burnett M, Lochner C, Warwick J, Dupont P, et al (2010): Changes in regional brain volumes in social anxiety disorder following 12 weeks of treatment with escitalopram. Metab Brain Dis 25:369-374

24. Roy-Byrne PP, Craske MG, Stein MB (2006): Panic disorder. Lancet 368 : 1023-1032.

25. Weissman MM (1993): Family genetic studies of panic disorder. J Psychiatr Res 27(suppl 1):69-78.

26. Schneier FR, Johnson J, Hornig CD, Liebowitz MR, Weissman MM (1992): Social phobia. Comorbidity and morbidity in an epidemiologic sample. Arch Gen Psychiatry 49:282-288.

27. Kendler KS, Walters EE, Neale MC, Kessler RC, Heath AC, Eaves LJ (1995): The structure of the genetic and environmental risk factors for six major psychiatric disorders in women. Phobia, generalized anxiety disorder, panic disorder, bulimia, major depression, and alcoholism. Arch Gen Psychiatry 52:374-383.

28. Goldstein RB, Wickramaratne PJ, Horwath E, Weissman MM (1997): Familial aggregation and phenomenology of 'early'-onset (at or before age 20 years) panic disorder. Arch Gen Psychiatry 54:271-278.

29. Talati A, Ponniah K, Strug LJ, Hodge SE, Fyer AJ, Weissman MM (2008): Panic disorder, social anxiety disorder, and a possible medical syndrome previously linked to chromosome 13. Biol Psychiatry 63:594601.

30. Fyer A, Endicott, J, Mannuzza S, Klein DF (1985): Schedule for Affective Disorders and Schizophrenia-Lifetime Version, Modified for the Study of Anxiety Disorders (SADS-LA). New York: Anxiety Disorders Clinic, New York State Psychiatric Institute.

31. Weissman MM, Wickramaratne $P$, Adams $P$, Wolk S, Verdeli H, Olfson $M$ (2000): Brief screening for family psychiatric history: The family history screen. Arch Gen Psychiatry 57:675-682.

32. Leckman JF, Sholomskas D, Thompson WD, Belanger A, Weissman MM (1982): Best estimate of lifetime psychiatric diagnosis: A methodological study. Arch Gen Psychiatry 39:879-883.

33. Spielberger C, Gorsuch, RL, Lushene, RE (1970): Manual for the State-Trait Anxiety Inventory. Palo Alto, CA: Consulting Psychologists Press.

34. First MB, Spitzer RL, Gibbon M, Willians JBW (1997): Structured Clinical Interview for DSM-IV Axis 1 Disorders. Washington, DC: American Psychiatric Press.
35. Liebowitz MR (1987): Social phobia. Mod Probl Pharmacopsychiatry 22: 141-173.

36. Ashburner J, Friston KJ (2000): Voxel-based morphometry-the methods. Neuroimage 11:805-821.

37. Ashburner J, Friston KJ (2001): Why voxel-based morphometry should be used. Neuroimage 14:1238-1243.

38. Ashburner J, Friston KJ (2005): Unified segmentation. Neuroimage 26: 839-851.

39. Ashburner J (2007): A fast diffeomorphic image registration algorithm. Neuroimage 38:95-113.

40. Keller SS, Wilke M, Wieshmann UC, Sluming VA, Roberts N (2004): Comparison of standard and optimized voxel-based morphometry for analysis of brain changes associated with temporal lobe epilepsy. Neuroimage 23:860-868.

41. Mikl M, Marecek R, Hlustik P, Pavlicova M, Drastich A, Chlebus $P$, et al. (2008): Effects of spatial smoothing on fMRI group inferences. Magn Reson Imaging 26:490-503.

42. Lazaro L, Castro-Fornieles J, Cullell C, Andres S, Falcon C, Calvo R, et al. (2011): A voxel-based morphometric MRI study of stabilized obsessivecompulsive adolescent patients. Prog Neuropsychopharmacol Biol Psychiatry 35:1863-1869.

43. Yoo HK, Kim MJ, Kim SJ, Sung YH, Sim ME, Lee YS, et al. (2005): Putaminal gray matter volume decrease in panic disorder: An optimized voxelbased morphometry study. Eur J Neurosci 22:2089-2094.

44. Henley SM, Ridgway GR, Scahill RI, Kloppel S, Tabrizi SJ, Fox NC, et al. (2010): Pitfalls in the use of voxel-based morphometry as a biomarker: Examples from huntington disease. AJNR Am J Neuroradiol 31:711-719.

45. Silver M, Montana G, Nichols TE (2011): False positives in neuroimaging genetics using voxel-based morphometry data. Neuroimage 54:9921000.

46. Hayasaka S, Phan KL, Liberzon I, Worsley KJ, Nichols TE (2004): Nonstationary cluster-size inference with random field and permutation methods. Neuroimage 22:676-687.

47. Warwick JM, Carey P, Jordaan GP, Dupont P, Stein DJ (2008): Resting brain perfusion in social anxiety disorder: A voxel-wise whole brain comparison with healthy control subjects. Prog Neuropsychopharmacol Biol Psychiatry 32:1251-1256.

48. Kilts CD, Kelsey JE, Knight B, Ely TD, Bowman FD, Gross RE, et al. (2006): The neural correlates of social anxiety disorder and response to pharmacotherapy. Neuropsychopharmacology 31:2243-2253.

49. Baldacara L, Borgio JG, Lacerda AL, Jackowski AP (2008): Cerebellum and psychiatric disorders. Rev Bras Psiquiatr 30:281-289.

50. Goldin PR, Manber T, Hakimi S, Canli T, Gross JJ (2009): Neural bases of social anxiety disorder: Emotional reactivity and cognitive regulation during social and physical threat. Arch Gen Psychiatry 66:170-180.

51. Straube T, Kolassa IT, Glauer M, Mentzel HJ, Miltner WH (2004): Effect of task conditions on brain responses to threatening faces in social phobics: An event-related functional magnetic resonance imaging study. Biol Psychiatry 56:921-930.

52. Polosan M, Baciu M, Cousin E, Perrone M, Pichat C, Bougerol T (2011): An fMRI study of the social competition in healthy subjects. Brain Cogn 77:401-411.

53. Fairhall SL, Ishai A (2007): Effective connectivity within the distributed cortical network for face perception. Cereb Cortex 17:2400-2406.

54. Gentili C, Gobbini MI, Ricciardi E, Vanello N, Pietrini P, Haxby JV, et al. (2008): Differential modulation of neural activity throughout the distributed neural system for face perception in patients with Social Phobia and healthy subjects. Brain Res Bull 77:286-292.

55. Lai CH (2011): Gray matter deficits in panic disorder: A pilot study of meta-analysis. J Clin Psychopharmacol 31:287-293.

56. Massana G, Serra-Grabulosa JM, Salgado-Pineda P, Gasto C, Junque C, Massana J, et al. (2003): Parahippocampal gray matter density in panic disorder: A voxel-based morphometric study. Am J Psychiatry 160:566568.

57. Uchida RR, Del-Ben CM, Busatto GF, Duran FL, Guimaraes FS, Crippa JA, et al. (2008): Regional gray matter abnormalities in panic disorder: A voxel-based morphometry study. Psychiatry Res 163:21-29.

58. Zahn R, Moll J, Krueger F, Huey ED, Garrido G, Grafman J (2007): Social concepts are represented in the superior anterior temporal cortex. Proc Natl Acad Sci U S A 104:6430-6435.

59. Zahn R, Moll J, Paiva M, Garrido G, Krueger F, Huey ED, et al. (2009): The neural basis of human social values: Evidence from functional MRI. Cereb Cortex 19:276-283. 
60. Liu W, Miller BL, Kramer JH, Rankin K, Wyss-Coray C, Gearhart R, et al. (2004): Behavioral disorders in the frontal and temporal variants of frontotemporal dementia. Neurology 62:742-748.

61. Tillfors M, Furmark T, Marteinsdottir I, Fredrikson M (2002): Cerebra blood flow during anticipation of public speaking in social phobia: A PET study. Biol Psychiatry 52:1113-1119.

62. Meda SA, Pryweller JR, Thornton-Wells TA (2012): Regional brain differences in cortical thickness, surface area and subcortical volume in individuals with Williams syndrome. PLoS One 7:e31913.

63. Reiss AL, Eckert MA, Rose FE, Karchemskiy A, Kesler S, Chang M, et al. (2004): An experiment of nature: brain anatomy parallels cognition and behavior in Williams syndrome. J Neurosci 24:5009-5015.

64. Blackmon K, Barr WB, Carlson C, Devinsky O, Dubois J, Pogash D, et al. (2011): Structural evidence for involvement of a left amygdala-orbitofrontal network in subclinical anxiety. Psychiatry Res 194:296-303.

65. Milad MR, Rauch SL (2007): The role of the orbitofrontal cortex in anxiety disorders. Ann N Y Acad Sci 1121:546-561.

66. Baur V, Bruhl AB, Herwig U, Eberle T, Rufer M, Delsignore A, et al. (2011): Evidence of frontotemporal structural hypoconnectivity in social anxiety disorder: A quantitative fiber tractography study [published online ahead of print November 11]. Hum Brain Mapp.

67. Baur V, Hanggi J, Rufer M, Delsignore A, Jancke L, Herwig U, et al. (2011): White matter alterations in social anxiety disorder. J Psychiatr Res 45 $1366-1372$.
68. Biederman J, Hirshfeld-Becker DR, Rosenbaum JF, Herot C, Friedman D, Snidman N, et al. (2001): Further evidence of association between behavioral inhibition and social anxiety in children. Am J Psychiatry 158: 1673-1679.

69. Schwartz CE, Snidman N, Kagan J (1999): Adolescent social anxiety as an outcome of inhibited temperament in childhood. J Am Acad Child Adolesc Psychiatry 38:1008-1015.

70. Schwartz CE, Kunwar PS, Greve DN, Moran LR, Viner JC, Covino JM, et al. (2010): Structural differences in adult orbital and ventromedial prefrontal cortex predicted by infant temperament at 4 months of age. Arch Gen Psychiatry 67:78-84.

71. Etkin A, Wager TD (2007): Functional neuroimaging of anxiety: A metaanalysis of emotional processing in PTSD, social anxiety disorder, and specific phobia. Am J Psychiatry 164:1476-1488.

72. Weissman MM, Brown AS, Talati A (2011): Translational epidemiology in psychiatry: Linking population to clinical and basic sciences. Arch Gen Psychiatry 68:600-608.

73. Davatzikos C (2004): Why voxel-based morphometric analysis should be used with great caution when characterizing group differences. Neuroimage 23:17-20.

74. Crum WR, Griffin LD, Hill DL, Hawkes DJ (2003): Zen and the art of medical image registration: Correspondence, homology, and quality. Neuroimage 20:1425-1437.

75. Ioannidis JP (2011): Excess significance bias in the literature on brain volume abnormalities. Arch Gen Psychiatry 68:773-780 